Proc. Indian Acad. Sci. (Earth Planet. Sci.), Vol. 90, No. 3, November 1981, pp. 197-209.

(C) Printed in India.

\title{
Characteristic curves for interpreting gravity anomalies of vertical cylinders and horizontal circular dises
}

\author{
C VISWESWARA RAO and I V RA.DHAKRISHNA MURTHY \\ Department of Geophysics, Andhra University, Waltair 530 003, India
}

MS received 24 April 1981

\begin{abstract}
Characteristic curves for the complete interpretation of a wide range of parameters of a verticá cylinder and a horizontal circular aisc are presented $\cdots$ It is established that the distance of the inflexion point on the gravity profile of a vertical cylinder from the point of maximum anomaly [i,e. the origin], is approximately fqual to the tadius of the vertical cylinder, thus enabling one to demarcate the boundary of the vertical cylinder directly from the contour máp based on the cluster of contours. Two gravity profiles, one across an anomalous zone in central Alberta, Canada and the other over Borsad area, India are interpreted by the curves presented and the results are shown.
\end{abstract}

Keywords. Gravity interpretation; characteristic curves; vertical cylinder; horizontal circular disc.

\section{Introduction}

It has been well established that interpretation of gravity and magnetic anomalies is greatly facilitated by the use of characteristic curves which provide information of the body parameters of the causative bodies very rapidly and without using computers. The method of characteristic curves has been used in one form or the other for a very long time in gravity and magnetic interpretation, but it is only in 1966 that the method has been put in to a systematic format (Grant and Martin 1966). Characteristic curves have been constructed for interpreting magnetic anomalies of individual geometric models by several workers (Smellie 1956; Hood 1964; Moo 1965; Bruckshaw and Kunaratnam 1963; Bean 1966; Grant and West 1965; Am 1972). Rao et al (1974), Rao and Murthy (1978) and Nageswara Rao et al (1979) have constructed characteristic curves for all geometric models "requently used in gravity and magnetic interpretation which make use of readily sheasurable distances and anomalies on the field profile. It has been observed by experience that these curves yield reliable results in the majority of cases. Some extreme cases of a vertical cylinder involving large radii could not, however, be satisfactorily interpreted with their curves. This drawback may be overcome 
if one uses the curves prepared with the characteristic distances involving the inflexion point and the points of one-third and two-thirds the maximum horizontal gradient. Such a set of characteristic curves for interpreting gravity anomalies of vertical circular cylinder are presented in this paper along with the relevant data to facilitate the interpreter to construct the charts to an elongated scale. Two extreme cases of vertical cylinder, viz., the circular disc and the bottomless circular cylinder are also considered. Some thumb rules helpful for rapid interpretation are also included.

\section{Vertical circular cylinder}

The vertical circular cylinder has three parameters, viz., $Z_{1}, Z_{2}$ and $R$, explained in figure 1 , to be interpreted from the shape of the profile, while the density contrast $(\sigma)$ can be determined from the maximum gravity anomaly. The three length parameters can be made into two dimensionless ratios $Z_{2} / Z_{1}$ and $R / Z_{1}$. The characteristic curves constructed for vertical cylinder are shown in figures 2,3 and 4 and the related data are presented in tables $1,2,3$ and 4 . All these data have been obtained using IBM 1130 computer available at Andhra University.

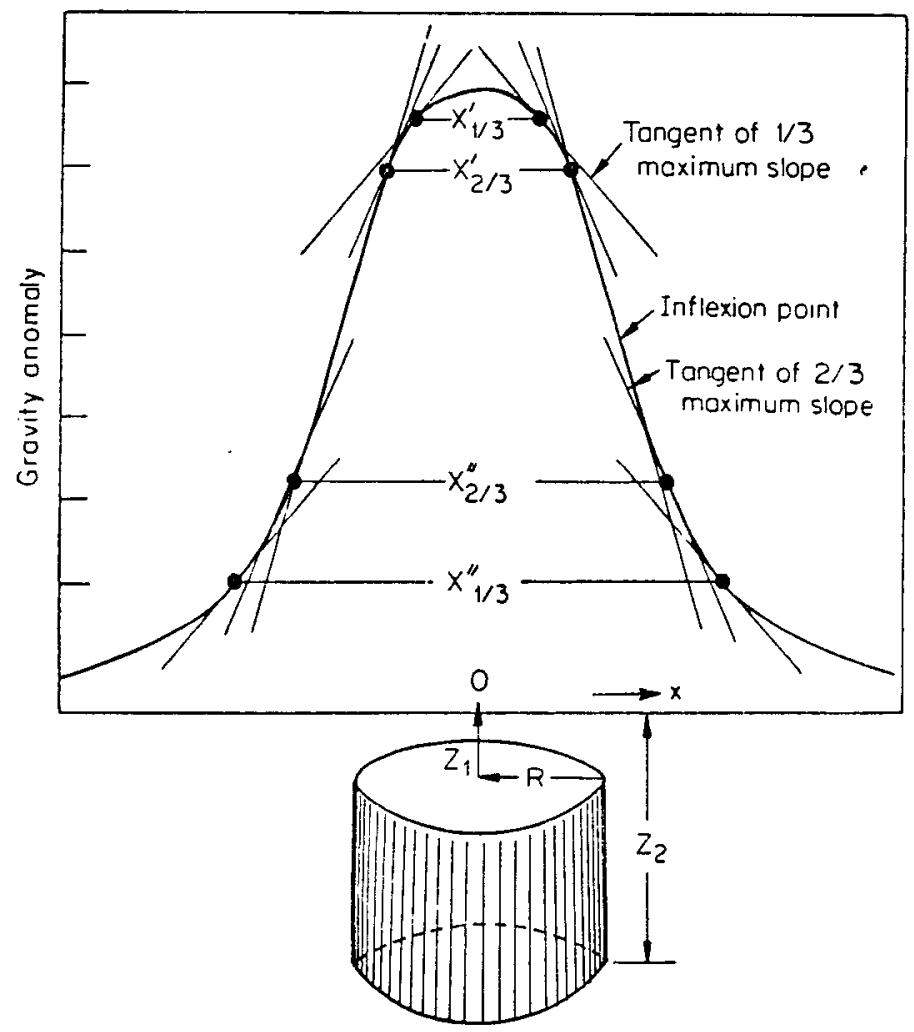

Figure 1. Parameters of vertical circular cylinder and characteristic distances used in ite interpretation. 


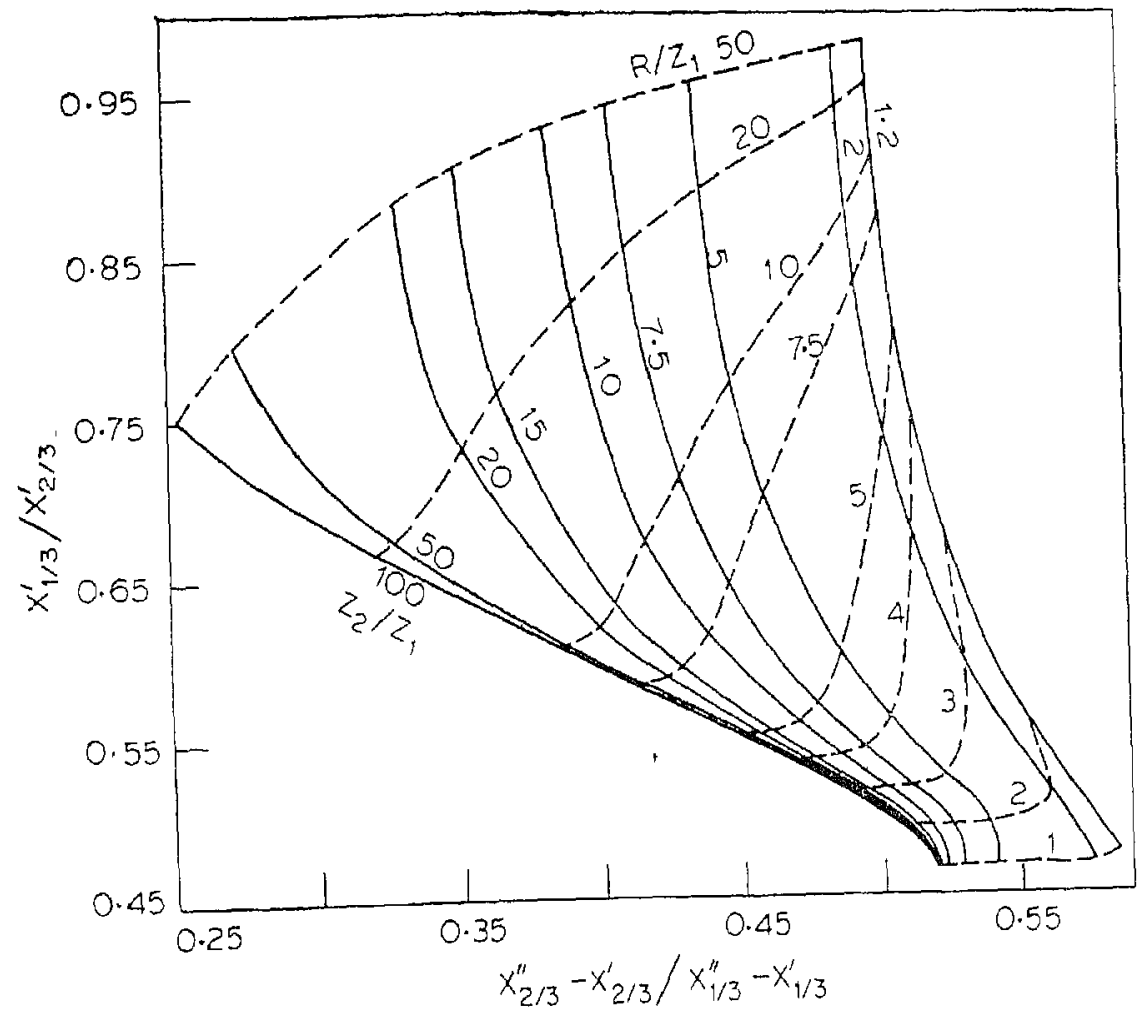

Figure 2. Characteristic curves for interpreting gravity anomalies of vertical cylinders.

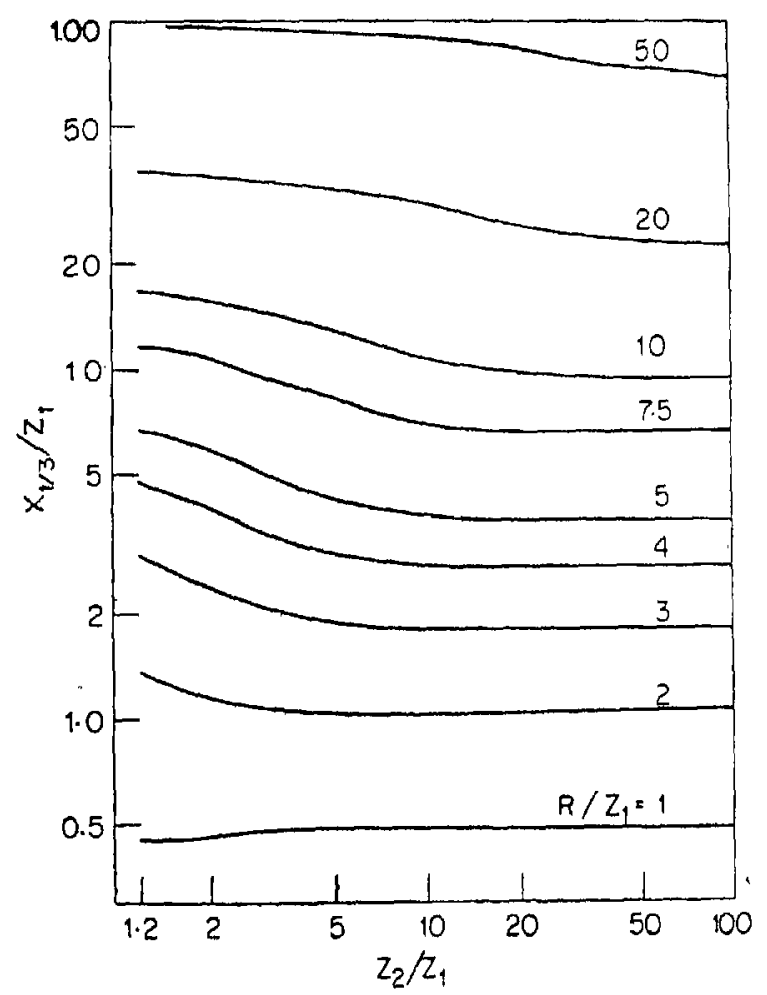

Figure 3. Complementary curves for interpreting gravity anomalies of vertical cylinders. 


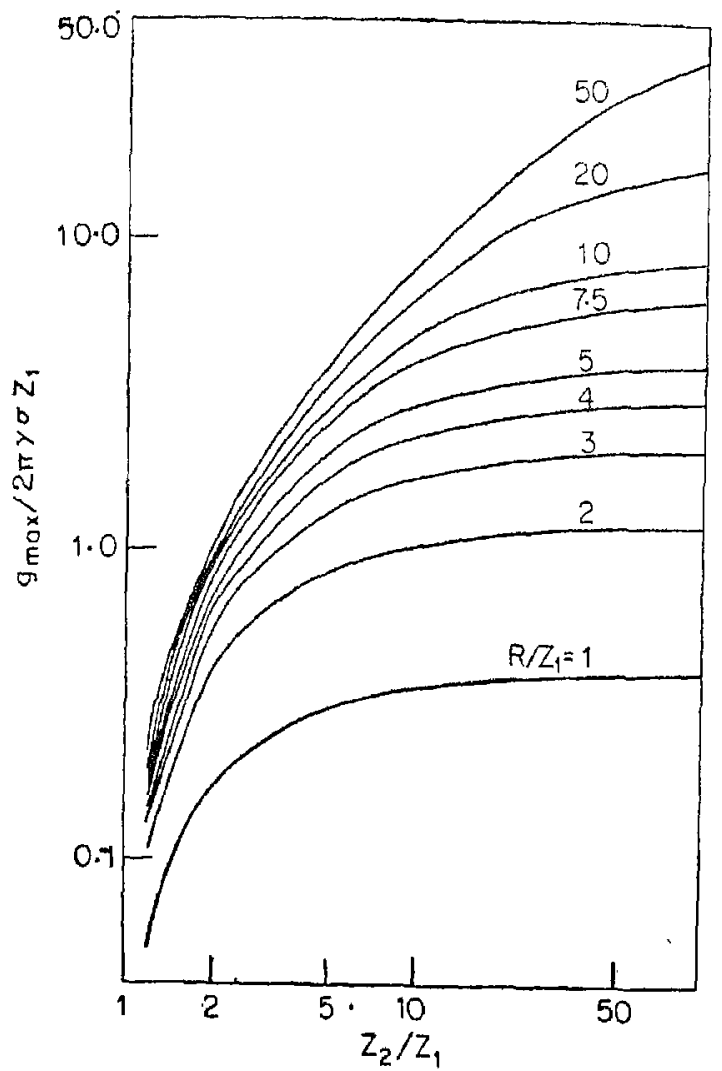

Figure 4. Complementary curves for determining the density contrast of the vertical cylinder.

Table 1. Data for the construction of characteristic curves in figure 2 .

\begin{tabular}{|c|c|c|c|c|c|c|c|c|c|}
\hline \multirow{2}{*}{$R / Z_{1}$} & \multicolumn{9}{|c|}{ The value of the ratio $X_{1 / 3}^{\prime} / X_{2 / 3}^{\prime}$ for $Z_{2} / Z_{1}$} \\
\hline & 1.2 & 2.0 & 5.0 & 7.5 & 10.0 & 15.0 & 20.0 & 50.0 & 100.0 \\
\hline 1.0 & 0.476 & 0.470 & 0.469 & 0469 & 0.468 & 0.468 & 0.469 & 0.469 & 0.469 \\
\hline 2.0 & 0.562 & 0.517 & 0.494 & 0.494 & 0.493 & 0.493 & 0.493 & 0.493 & 0.493 \\
\hline 3.0 & 0.676 & 0.598 & 0.525 & 0.518 & 0.517 & 0.516 & 0.516 & 0.516 & 0.516 \\
\hline 4.0 & 0.757 & 0.679 & 0.562 & 0.544 & 0.539 & 0.536 & 0.536 & 0.536 & 0536 \\
\hline 5.0 & 0.810 & 0.742 & 0,604 & 0.571 & 0.561 & 0.555 & 0.553 & 0.552 & 0.552 \\
\hline 7.5 & 0.879 & 0.834 & 0.703 & 0.645 & 0.617 & 0.595 & 0.589 & 0.584 & 0.584 \\
\hline 10.0 & 0.913 & 0.880 & 0.774 & 0.713 & 0.673 & 0.635 & 0.621 & 0.609 & 0.608 \\
\hline 20.0 & 0.958 & 0.944 & 0.892 & 0.854 & 0.821 & 0.768 & 0.732 & 0674 & 0.667 \\
\hline 50.0 & 0.984 & 0.978 & 0.959 & 0.945 & 0.932 & 0.907 & 0.884 & 0.786 & 0.751 \\
\hline
\end{tabular}

These characteristic curves involve (i) $X^{\prime}{ }_{1 / 3}$ and $X^{\prime \prime}{ }_{1 / 3}$, the distances between two sets of points of one-third the maximum horizontal gradient, one set near the origin-the point of maximum gravity anomaly, and the other far away from 
Table 2. Data for the construction of characteristic curves in figure 2 .

\begin{tabular}{rrrrrrrrrrrr}
\hline$R / Z_{1}$ & \multicolumn{7}{c}{ The values of the ratio $\left(X^{\prime \prime}{ }_{2 / 3}-X^{\prime}{ }_{2 / 3}\right) /\left(X^{\prime \prime}{ }_{1 / 3}-X_{1 / 3}^{\prime}\right)$ for $Z_{2} / Z_{1}$} \\
\hline & 1.2 & 2.0 & 5.0 & 7.5 & 10.0 & 15.0 & 20.0 & 50.0 & 100.0 \\
\hline 1.0 & 0.586 & 0.576 & 0.541 & 0.529 & 0.523 & 0.520 & 0.519 & 0.518 & 0.518 \\
2.0 & 0.552 & 0.560 & 0.539 & 0.525 & 0.517 & 0.511 & 0.509 & 0.508 & 0.508 \\
3.0 & 0.524 & 0.531 & 0.522 & 0.510 & 0.502 & 0.494 & 0.491 & 0.488 & 0.488 \\
4.0 & 0.513 & 0.512 & 0.503 & 0.493 & 0.485 & 0.476 & 0.472 & 0.468 & 0.467 \\
5.0 & 0.508 & 0.503 & 0.485 & 0.477 & 0.470 & 0.460 & 0.455 & 0.449 & 0.449 \\
7.5 & 0.503 & 0.494 & 0.460 & 0.446 & 0.438 & 0.428 & 0.422 & 0.414 & 0.413 \\
10.0 & 0.501 & 0.491 & 0.449 & 0.429 & 0.418 & 0.406 & 0.399 & 0.387 & 0.385 \\
20.0 & 0.500 & 0.488 & 0.439 & 0.412 & 0.392 & 0.367 & 0.352 & 0.327 & 0.322 \\
50.0 & 0.499 & 0.487 & 0.437 & 0.407 & 0.384 & 0.352 & 0.330 & 0.273 & 0.252 \\
\hline
\end{tabular}

Table 3. Data for the construction of complementary curves in figure 3.

\begin{tabular}{rrrrrrrrrr}
\hline & \multicolumn{8}{c}{ The values of the ratio $X^{\prime} 1 / 3 / Z_{1}$ for $Z_{2} / Z_{1}$} \\
\cline { 2 - 10 } & \multicolumn{1}{c}{1.2} & 2.0 & 5.0 & \multicolumn{1}{c}{7.5} & 10.0 & 15.0 & 20.0 & 50.0 & 100.0 \\
\hline 1.0 & 0.454 & 0.458 & 0.482 & 0.485 & 0.486 & 0.487 & 0.487 & 0.487 & 0.487 \\
2.0 & 1.361 & 1.137 & 1.045 & 1.047 & 1.049 & 1.050 & 1.051 & 1.051 & 1.051 \\
3.0 & 2.920 & 2.356 & 1.865 & 1.827 & 1.821 & 1.819 & 1.819 & 1.819 & 1.819 \\
4.0 & 4.780 & 3.988 & 2.938 & 2.782 & 2.740 & 2.722 & 2.718 & 2.718 & 2.718 \\
5.0 & 6.748 & 5.834 & 4.256 & 3.908 & 3.792 & 3.730 & 3.716 & 3.708 & 3.708 \\
7.5 & 11.750 & 10.746 & 8.358 & 7.418 & 6.966 & 6.628 & 6.526 & 6.456 & 6.452 \\
10.0 & 16.770 & 15.754 & 13.050 & 11.668 & 10.822 & 10.020 & 9.720 & 9.468 & 9.452 \\
20.0 & 36.820 & 35820 & 32.920 & 31.040 & 29.460 & 27.120 & 25.620 & 22.140 & 22.840 \\
50.0 & 96.860 & 95.880 & 93.060 & 91.120 & 89.400 & 86.400 & 83.800 & 74.160 & 69.480 \\
\hline
\end{tabular}

Table 4. Data for the construction of complementary curves in figure 4.

\begin{tabular}{|c|c|c|c|c|c|c|c|c|c|}
\hline \multirow{2}{*}{$R / Z_{1}$} & \multicolumn{9}{|c|}{ The values of the function $g_{\max } / 2 \pi \gamma \sigma Z_{1}$ for $Z_{2} / Z_{1}$} \\
\hline & 1.2 & 2.0 & 5.0 & 7.5 & 10.0 & 150 & 20.0 & 50.0 & 100.0 \\
\hline 1.0 & 0.052 & 0.178 & 0.315 & 0.348 & 0.364 & 0.381 & 0.389 & 0.404 & 0.409 \\
\hline 2.0 & 0.103 & 0.407 & 0.851 & 0.974 & 1.038 & 1.103 & 1.136 & 1.196 & 1.216 \\
\hline 3.0 & 0.113 & 0.557 & 1.331 & 1.584 & 1.722 & 1.865 & 1.938 & 2.072 & 2.117 \\
\hline 4.0 & 0.147 & 0.651 & 1.719 & 2.123 & 2.353 & 2.598 & 2.727 & 2.963 & 3.043 \\
\hline 5.0 & 0.157 & 0.714 & 2.028 & 2.585 & 2.918 & 3.287 & 3.483 & 3.849 & 3.974 \\
\hline 7.5 & 0.171 & 0.804 & 2.552 & 3.459 & 4.066 & 4.796 & 5.206 & 6.007 & 6.288 \\
\hline 10.0 & 0.178 & 0.851 & 2.869 & 4.049 & 4.908 & 6.022 & 6.689 & 8.059 & 8.551 \\
\hline 20.0 & 0.189 & 0.925 & 3.409 & 5.165 & 6.664 & 9.025 & 10.741 & 15.173 & 17.044 \\
\hline 50.0 & 0.195 & 0.970 & 3.761 & 5.950 & 8.019 & 11.808 & 15.158 & 28.299 & 37.206 \\
\hline
\end{tabular}


it, and (ii) $X_{2}^{\prime} / 3$ and $X_{2 / 3}^{\prime \prime}$, the distances between two sets of points of twothird the maximum horizontal gradient, one set near the origin and the other far away from it. These distances are explained in figure 1 . Figure 2 gives the ratios $Z_{2} / Z_{1}$ and $R / Z_{1}$, while figures 3 and 4 provide information on $Z_{1}$ and the density contrast respectively.

The gravity effect $(g)$ at a distance $x$ from the origin of a vertical cylinder, extending from depth $Z_{1}$ to depth $Z_{2}$, is obtained by subtracting the gravity effect of the vertical cylinder whose depth to the top is $Z_{2}$ from the gravity effect of the vertical cylinder whose depth to the top is $Z_{1}$ (Nabhigian 1962). The relevant equation is given by

$$
\begin{aligned}
g(x)=2_{3}^{y} \gamma \sigma & {\left[\frac{R^{2}-x^{2}}{\sqrt{(x+R)^{2}+Z_{1}^{2}}} K(k)+\sqrt{(x+R)^{2}+Z_{2_{1}^{2}}} E(k)\right.} \\
& -\frac{R^{2}-x^{2}}{\sqrt{(x+R)^{2}+Z_{2}^{2}}} K(k)-\sqrt{(x+R)^{2}+Z_{2}^{2}} E(k) \\
& \left.+\frac{\pi\left(Z_{1}-Z_{2}\right)}{2} \wedge(\phi, k)-\pi\left(Z_{1}-Z_{2}\right)\right]
\end{aligned}
$$

where $K(k)$ is the complete elliptic integral of the first kind, $E(k)$ is the " complete elliptic integral of the second kind, $\wedge(\phi, k)$ is the Neumann's Lambda function,

$$
k=\sqrt{\frac{4 x R}{(x+R)^{2}+Z^{2}}}=\text { the modulus of the elliptic integrals, }
$$

$\sigma$ is the density contrast, and $\gamma$ is the gravitational constant. The maximum gravity anomaly is given by

$$
g_{\max }=g(0)=2 \pi \gamma \sigma\left[Z_{2}-Z_{1}+\sqrt{Z_{1}^{2}+R^{2}}-\sqrt{Z_{2}^{2}+R^{2}}\right] .
$$

The first and second horizontal derivatives of gravity anomaly are obtained as

$$
\begin{aligned}
& \frac{\partial g}{\partial x}=2 \gamma \sigma R\left[-\frac{R^{2}+x^{2}+Z_{1}^{2}}{x \sqrt{(R+x)^{2}+Z_{1}^{2}}} K(k)+\frac{\sqrt{(R+x)^{2}+Z_{1}^{2}}}{x} E(k)\right. \\
& +\frac{R^{2}+x^{2}+Z_{2}^{2}}{\left.x \sqrt{(R+x)^{2}+Z_{2}^{2}} K(k)-\frac{\sqrt{(R+x)^{2}+Z_{2}^{2}}}{x} E(k)\right]} \\
& \quad \text { and } \frac{\partial^{2} g}{\partial x^{2}}=\frac{2 \gamma \sigma R^{2}}{x^{2}\left[(R-x)^{2}+Z_{1}^{2}\right] \sqrt{(R+x)^{2}+Z_{1}^{2}}} \\
& \quad \times\left[\left(R^{2}+Z_{1}^{2}\right)\left\{(R-x)^{2}+Z_{2}^{2}\right\} K(k)\right. \\
& \left.\quad-\left\{\left(Z_{2}^{2}+R^{2}\right)^{2}+x^{2}\left(Z_{2}^{2}-R^{2}\right)\right\} E(k)\right]
\end{aligned}
$$




$$
\begin{aligned}
& -\frac{2 y \sigma R^{2}}{x^{2}\left[(R-x)^{2}+Z_{j}\right] \sqrt{(R+x)^{2}+Z_{2}^{j}}} \\
& \times\left[\left(R^{2}+Z_{3}^{2}\right) \quad\left\{(R-x)^{2}+Z_{2}^{2}\right\} K(k)\right. \\
& \left.-\left\{\left(Z_{2}^{3}+R^{2}\right)^{2}+x^{2}\left(Z_{2}^{3}-R^{2}\right)\right\} E(k)\right]
\end{aligned}
$$

Equation 3, however, does not allow calculation of points of inflexion easily. Hence, the inflexion point is determined and other distances are calculated by numerical methods using (2) and (3) as follows:

Values of $Z_{2} / Z_{1}$ and $R / Z_{1}$ are first assumed. The first and second derivatives for different values of $x$ are then calculated at reasonable intervals of $x$ using (2) and (3). The values of $x$ where the second derivative is zero is the inflexion point. Hence two values of $x$ ( say $x_{1}$ and $x_{2}$ ) are determined from the calculated second derivatives, between which $x_{\text {in } 11}$ lies and the second derivative becomes zero. The exact value of $x_{\text {inf } 1}$ is determined by linear interpolation. If $D_{1}$ and $D_{2}$ are the second derivatives at $x_{1}$ and $x_{2}$ respectively, then a new point $x_{0}$ is located by the interpolation formula

$$
x_{0}=x_{1}+\frac{\left(x_{2}-x_{1}\right)}{\left(D_{1}-D_{2}\right)} D_{1} \text {. }
$$

The second derivative value $D_{0}$ is then calculated at $x_{0}$ by (3). It may be noted that $D_{1}$ is necessarily negative, while $D_{2}$ is positive. If $D_{0}$ is negative, further interpolation is performed between $x_{0}$ (treating it as $x_{1}$ ) and $x_{2}$. If $D_{0}$ is positive, interpolation is performed between $x_{1}$ and $x_{0}$ (treating it as $x_{2}$ ). This procedure is repeated several times.

The maximum horizontal gradient is then calculated at $x_{\mathrm{in} \theta}$ using (2). Two values of $x$ at which the gradient is nearer to one-third the maximum gradient and two more values of $x$ at which the gradient is nearer to two-thirds the maximum gradient are then located and improved by Newton-Raphson's method. The ratio $g_{\max } / 2 \pi \gamma \sigma Z_{1}$ for figure 4 is obtained from (1).

The characteristic curves presented in figures 2,3 and 4 are used in the following way:

From the field profile, we find out the distances $X_{1 / 3}^{\prime}, X_{2 / 3}^{\prime}, X_{2 / 3}^{\prime \prime}, X_{1 / 3}^{\prime \prime}$, and then calculate the estimators $\left(X^{\prime \prime}{ }_{2 / 3}-X_{2 / 3}^{\prime}\right) /\left(X^{\prime \prime}{ }_{1 / 3}-X_{1 / 3}^{\prime}\right)$ and $\left(X_{1 / 3}^{\prime} /\right.$ $\left.X_{2 / 3}^{\prime}\right)$ to determine $Z_{2} \mid Z_{1}$ and $R / Z_{1}$ from figure 2 . Figure 3 gives the values of $X_{1 / 3}^{\prime} / Z_{1}$ for the known values of $Z_{2} / Z_{1}$ and $R / Z_{1}$. Since $X_{1 / 3}^{\prime}$ is known from the field profile, $Z_{1}$ and hence $Z_{2}$ and $R$ are calculated. Figure 4 provides the value of the function $g_{\max } / 2 \pi \gamma \sigma Z_{1}$ for the known values of $Z_{2} / Z_{1}$ and $R / Z_{1} . \quad g_{\max }$ is measured from the field profile and is compared with this function to determine density contrast.

\subsection{Some thumb-rules}

It is of interest to note the dependence of $X_{\text {inn } 11} / Z_{1}$ on $R / Z_{1}$ and $Z_{2} / Z_{1}$ which is shown in table 5. It may be observed that if $R>Z_{1}, X_{\text {infl }}$ is less than $R$. 
Table 5. Dependence of the ratio $X_{\mathrm{inf}} / Z_{1}$ on $R / Z_{1}$ and $Z_{2} / Z_{1}$ of a vertical cylinder.

\begin{tabular}{rrrrrrrrrrr}
\hline$R / Z_{1}$ & \multicolumn{8}{c}{ The values of $X_{\text {inf } /} / Z_{1}$ for $Z_{2} / Z_{1}$} \\
\cline { 2 - 10 } & 1.2 & 2.0 & 5.0 & 7.5 & 10.0 & 15.0 & 20.0 & 50.0 & 100.0 \\
\hline 1.0 & 0.983 & 1.040 & 1.115 & 1.125 & 1.127 & 1.129 & 1.129 & 1.129 & 1.129 \\
2.0 & 1.916 & 1.911 & 1.954 & 1.969 & 1.976 & 1.979 & 1.981 & 1.982 & 1.982 \\
3.0 & 2.925 & 2.902 & 2.907 & 1.291 & 2.929 & 2.935 & 2.937 & 2.938 & 2.938 \\
4.0 & 3.937 & 3.913 & 3.896 & 3.904 & 3.912 & 3.919 & 3.921 & 3.924 & 3.924 \\
5.0 & 4.947 & 4.924 & 4.898 & 4.901 & 4.906 & 4.913 & 4.916 & 4.919 & 4.920 \\
7.5 & 7.462 & 7.444 & 7.414 & 7.409 & 7.409 & 7.413 & 7.416 & 7.421 & 7.422 \\
10.0 & 9.971 & 9.956 & 9.929 & 9.921 & 9.918 & 9.919 & 9.921 & 9.927 & 9.928 \\
20.0 & 19.985 & 19.977 & 19.960 & 19.953 & 19.948 & 19.944 & 19.943 & 19.945 & 19.947 \\
50.0 & 49.994 & 49.991 & 49.983 & 49.979 & 49.977 & 49.974 & 49.972 & 49.968 & 49.969 \\
\hline
\end{tabular}

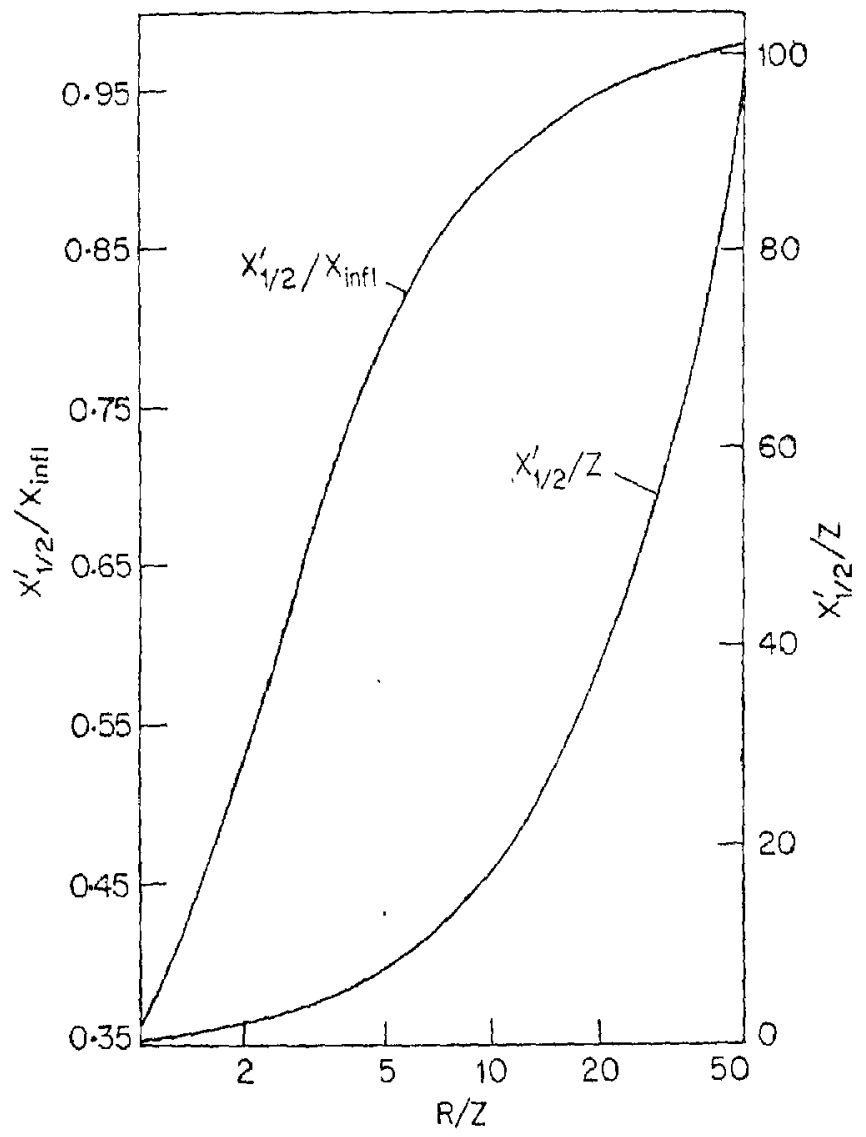

Figure 5. Characteristic and complementary curves for interpreting gravity anomalies of borizontal circular discs, 
Further, $X_{\text {inf1 }}$ decreases first and then increases with increase in $Z_{2}$. The initial decrease of $X_{\text {infi }}$ in case of small ratios of $Z_{2} / Z_{1}$ is not brought out by the contents of table 5 , because such a decrease might occur at values of $Z_{2}<1.2 Z_{1}$. For all practical purposes, however, $X_{\text {infl }}$ is approximately equal to $R$ and hence determines the radius of the cylinder. The value of $R$, thus obtained, is always an underestimate, the maximum error being not more than $3 \%$. For shallow cylindrical bodies, the inflexion point can be easily determined, because at these points the anomaly contours are crowded. Thus, the boundary of the vertical cylinder can be easily determined straight from the contour map based on the cluster of contours.

\section{Horizontal circular disc}

The horizontal circular disc may be viewed as a vertical cylinder whose $Z_{2} / Z_{1}$ ratio has a very small value. The exact expression for the gravity anomaly of this model is given by (Rao and Murthy 1966, 1978)

$$
g(x)=2 \gamma \sigma d Z\left[\pi-\frac{Z}{(x+R)^{2}+Z^{2}} K(k)-\frac{\pi}{2} \wedge(\phi, k)\right],
$$

where $Z$ is the depth to the centre of the disc and other symbols carry the meaning as for the vertical cylinder.

The characteristic distances used in this case are $X_{1 / 2}^{\prime}$, the distance between two points, nearer to the origin of half maximum gradient and $X_{\text {infl }}$, the distance between two points of inflexion gradient. These are calculated and their ratios determined for different values of $R / Z$ following the same procedure outlined in the case of vertical cylinders. Figure 5 shows the characteristic and complementary curves for determining $R$ and $Z$ from the gravity anomalies of horizontal circular discs. The data for the construction of figure 5 are given in table 6 . Figure 5 is used in the following way :

Table 6. Data for the construction of characteristic curves for circular disc.

\begin{tabular}{rrr}
\hline$R / Z$ & $X_{1 / 2 / X_{\text {inf }}}$ & $X^{\prime}{ }_{1 / 2} / Z$ \\
\hline 1.0 & 0.3616 & 0.7016 \\
2.0 & 0.5267 & 2.0240 \\
3.0 & 0.6602 & 3.8720 \\
4.0 & 0.7414 & 5.8500 \\
5.0 & 0.7927 & 7.8540 \\
7.5 & 0.8623 & 12.8780 \\
10.0 & 0.8971 & 17.8980 \\
20.0 & 0.9489 & 37.9200 \\
50.0 & 0.9797 & 97.9600 \\
\hline
\end{tabular}


The characteristic distances $X_{1 / 2}^{\prime}$ and $X_{\mathrm{in} 11}$ are measured from the field profile. The ratio $X_{1 / 2}^{\prime} / X_{\text {int? }}$ is calculated. The values of $R / Z$ and $X_{1 / 2}^{\prime} / Z$ are noted from figure 5. Since $X_{1 / 2}^{s}$ is known from the field profile, $Z$ and $R$ are calculated.

When $R$ and $Z$ are known, the surface density, $\sigma d Z$ of the circular disc can be calculated from the maximum gravity anomaly as follows :

$$
\sigma d Z=\frac{g_{\max }}{2 \pi y\left(1-\frac{Z}{\sqrt{R^{2}+Z^{2}}}\right)}
$$

where $d Z$ is the thickness of the circular disc. $\sigma$ and $d Z$ cannot be separated. One of them can be calculated if the other is known.

\section{Bottomless vertical cylinder}

When the depth $Z_{2}$ of the vertical cylinder has a very high value, the gravity anomaly is given by the equation (Nabhigian 1962)

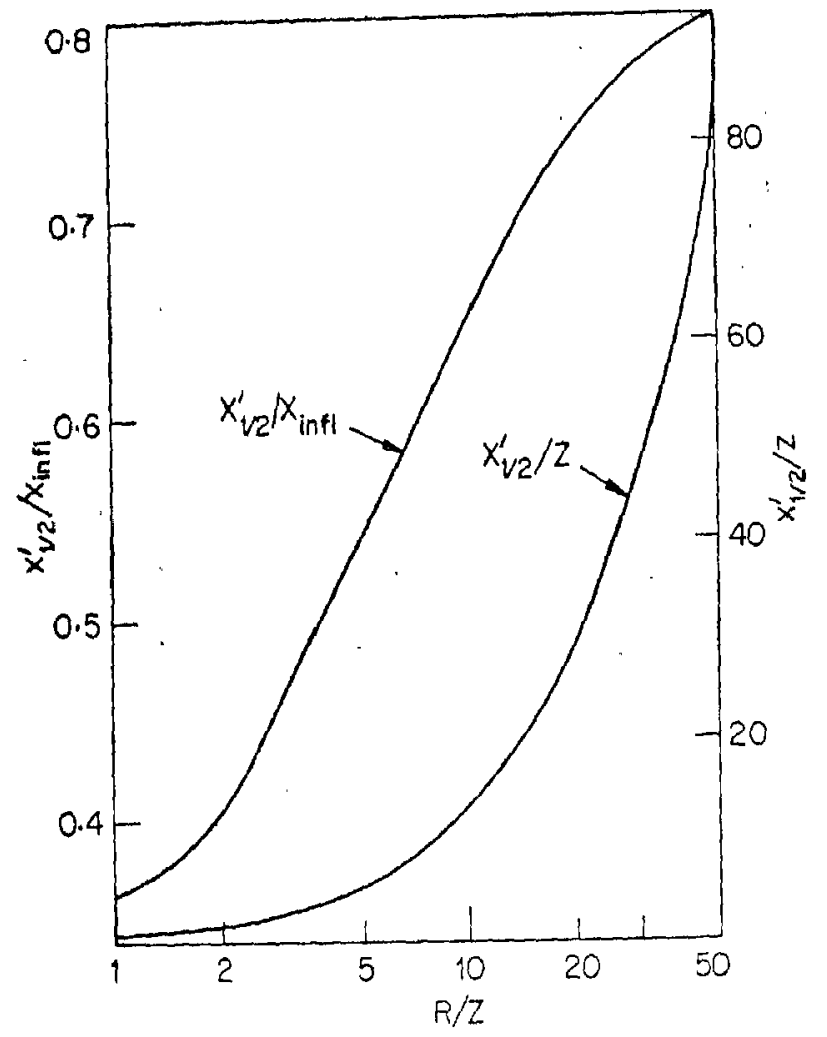

Figure 6. Characteristic and complementary curves for interpreting gravity anomalies of bottomless vertical cylinder. 


$$
\begin{gathered}
g(x)=2 \gamma \sigma\left[\frac{R^{2}-x^{2}}{\sqrt{(x+R)^{2}+Z^{2}}} K(k)+\sqrt{(x+R)^{2}+Z^{2}} E(k)\right. \\
\left.+\frac{\pi Z}{2} \wedge(\phi, k)-\pi Z\right],
\end{gathered}
$$

where $Z$ is the depth to the top of the bottomless vertical cylinder. The characteristic and complementary curves for this model are presented in figure 6 and the relevant data are given in table 7.

\section{Examples of interpretation}

The characteristic, curves presented in this paper have been used to interpret some field profiles. In general, the accuracy of interpretation depends on how accurately the inflexion and other points are located.

Example 1: Figure 7 shows the gravity profile across an anomalous zone in central Alberta (Skeels 1963) and its interpretation making use of charateristic curves of a vertical cylinder. The distances measured are given below.

$$
\begin{aligned}
& X_{1 / 3}^{\prime}=1.92 \mathrm{~km}, \quad X^{\prime \prime}{ }_{1 / 3}=16.80 \mathrm{~km} \\
& X_{2 / 3}^{\prime}=3.50 \mathrm{~km} \text { and } X^{\prime \prime}{ }_{2 / 3}=11.68 \mathrm{~km} .
\end{aligned}
$$

The characteristic estimators are calculated as

$$
X_{1 / 3}{ }_{1 / 3} / X_{2 / 3}^{\prime}=0.545 \text { and }\left(X^{\prime \prime \prime}{ }_{2 / 3}-X_{2 / 3}^{\prime}\right) /\left(X^{\prime \prime}{ }_{1 / 3}-X_{1 / 3}^{\prime}{ }_{1 / 3}\right)=0.548
$$

These values when located in figure 2 define the values of $Z_{2} \mid Z_{1}=2.1$ and $R / Z_{1}=2 \cdot 25$. From figure 3 , the values of $X_{1 / 3}^{\prime} / Z_{1}$. is found to be $1 \cdot 3$ corresponding to these values of $Z_{2} / Z_{1}$ and $R / Z_{1}$. Since $X_{1 / 3}^{\prime}=1.92 \mathrm{~km}$, $Z_{1}$ is calculated as $1.48 \mathrm{~km}$. From the known values of $Z_{1}, Z_{2} / Z_{1}$ and $R / Z_{1}$

Table 7. Data for the construction of characteristic curves for bottomless cylinder.

\begin{tabular}{ccc}
\hline$R / Z$ & $X^{\prime}{ }_{1 / 2} / X_{\text {inf }}$ & $X^{\prime}{ }_{1 / 2} / Z$ \\
\hline 1.0 & 0.3637 & 0.8308 \\
2.0 & 0.4075 & 1.6178 \\
3.0 & 0.4609 & 2.7100 \\
4.0 & 0.5052 & 3.9660 \\
5.0 & 0.5414 & 5.3280 \\
7.5 & 0.6073 & 9.0160 \\
10.0 & 0.6520 & 12.9440 \\
20.0 & 0.7467 & 29.7800 \\
50.0 & 0.8390 & 83.8400 \\
\hline
\end{tabular}




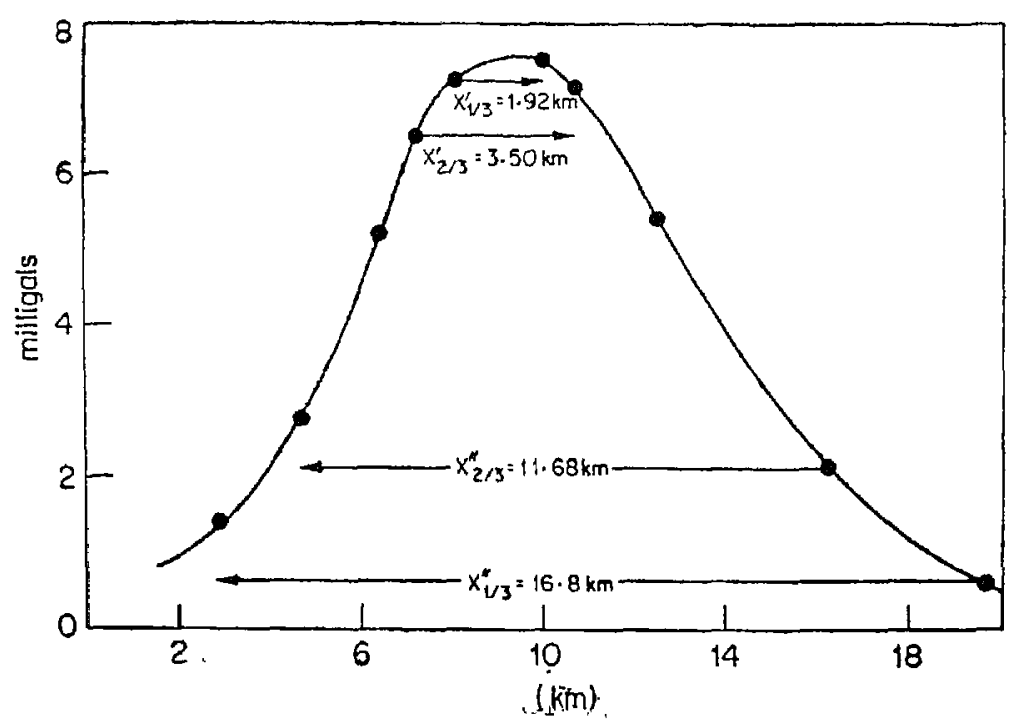

Figure 7. Interpretation of gravity anomaly profile in central Alberta [Skeels 1963].

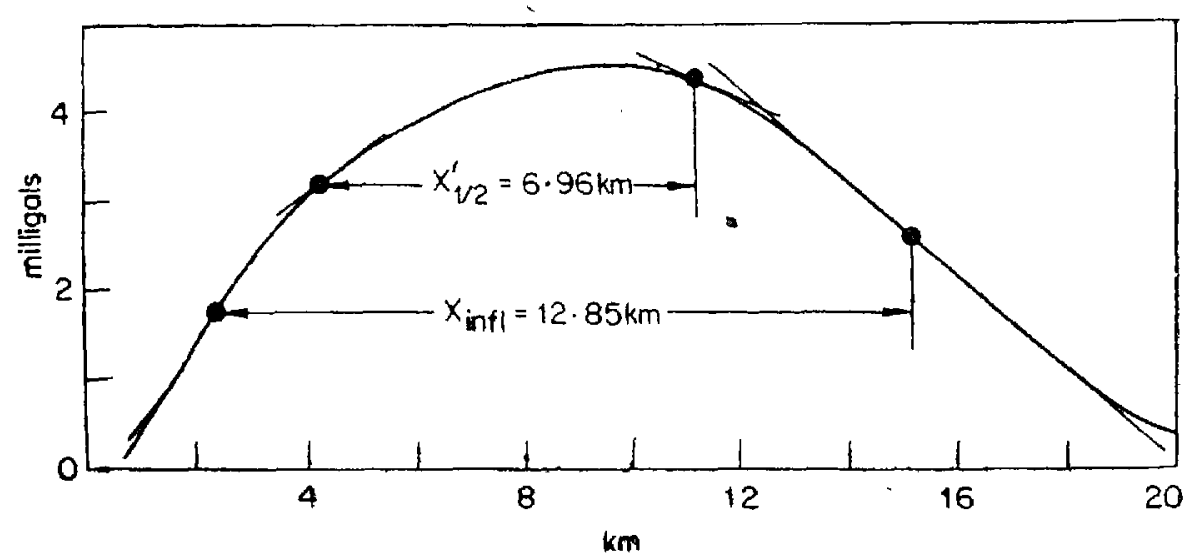

Figure 8. Interpretation of residual gravity anomaly profile over Borsad area, India [Rao and Murthy 1978].

the values of $Z_{2}$ and $R$ are calculated as $3.11 \mathrm{~km}$ and $3.33 \mathrm{~km}$ respectively. From figure 4 , it is obtained that $g_{\max } / 2 \pi y \sigma Z_{1}=0.46$. Since the value of $g_{\max }=-7.625 \mathrm{~m}$ gals, the density contrast $(\sigma)$ calculated is $-0.267 \mathrm{gm} / \mathrm{cc}$. The parameters obtained in this interpretation compare reasonably well with those obtained by Skeels $\left(Z_{1}=2.24 \mathrm{~km}, Z_{2}=3.74 \mathrm{~km}\right.$ and $\left.R=3.74 \mathrm{~km}\right)$ with his master curves assuming the density contrast to be $-0.3 \mathrm{gm} / \mathrm{cc}$. The difference in $Z_{1}$ is due to the fact that the density contrast is assumed to be $-0.30 \mathrm{gm} / \mathrm{cc}$ by Skeels, while in the present method it is solved from the field profile.

Example 2 : Figure 8 depicts the residual gravity anomaly profile over Borsad area, India, believed to be due to structure in the basement (Rao and Murthy, 
1978). This anomaly is interpreted using characteristic curves of horizontal circular disc. As the profile is not perfectly symmetric, the characteristic distances on either flank of the profile are measured. The distances thus measured are $X_{1 / 2}^{\prime}=6.96 \mathrm{~km}$ and $x_{\mathrm{infl}}=12.85 \mathrm{~km}$. The characteristic estimator is $X_{1 / 2}^{\prime} / x_{\text {in } 11}=0.5437$ which when located in figure 5 gives that $X_{1 / 2}^{\prime} / Z=2.4$ and $R / Z=2 \cdot 1$. Since, the value of $X_{1 / 2}^{\prime}=6.96 \mathrm{~km}$, the depth to the top $(Z)$ of the circular disc is calculated as $2.9 \mathrm{~km}$ and hence $R=6.1 \mathrm{~km}$. The calculated value of $Z$ roughly corresponds to the maximum thickness of sediments in this area.

\section{Acknowledgements}

Dr V Nageswara Rao was closely associated with this work and carried out lot of computational work.

\section{References}

Am K 1972 Geoexploration 1063

Bean R J 1966 Geophysics 31963

Bruckshaw J M and Kunaratnam K 1963 Geophys. Prospect. 11510

Grant F S and Martin L 1966 Geoplysics 31135

Grant F S and West G F 1965 Interpretation theory in applied Geoplysics (New York: McGraw Hill)

Hood P 1964 Geophys. Prospect. 12440

Moo J K C 1965 Geophys. Prospect. 13203

Nabhigian M N 1962 Geofisica Pura E Applicata 5345

Nageswara Rao V, Visweswara Rao C and Radhakrishna Murthy IV 1979 Geophys. Res. Bull. 1737

Rao B S R and Radhakrishna Murthy IV 1966 Indian J Pure Appl. Phys. 4276

Rao B S R and Radhakishna Murthy I V 1978 Gravity and magnetic methods of prospecting (New Delhi : Arnold Heinmann)

Rao B S R Radhaknishna Murthy I V and Visweswara Rao C 1974 Gravity interpretation by characteristic curves Monograph Series No. 118 Andhra University

Skeels D C 1963 Geophysics 28724

Smelli D W 1956 Geophys, 211021 